\title{
Inhibition of cAMP/PKA/CREB Signaling Axis Improves Fi- broblast Plasticity for Direct Cardiac Reprogramming
}

\author{
Emre Bektik ${ }^{1,2}$, Yu Sun ${ }^{3}$, Adrienne T. Dennis ${ }^{3}$, Phraew Sakon ${ }^{2}$, Dandan Yang ${ }^{1}$, and Jidong Fu 1,3, * \\ 1 Department of Physiology and Cell Biology College of Medicine, Ohio State University, 333 W 10th Ave \\ Columbus, OH 43210, the USA; Email: Emre.Bektik@childrens.harvard.edu or dandan.yang@osumc.edu \\ 2 Boston Children's Hospital, Harvard Medical School, 300 Longwood Avenue, Boston, MA 02115, USA; \\ Email: psakon@syr.edu \\ 3 Department of Medicine, Heart and Vascular Research Center, MetroHealth Campus, Case Western Reserve \\ University, Cleveland, 44109 OH, USA; Email: suny4@ccf.org or adennis@metrohealth.org \\ * Correspondence: Email: \idong.Fu@osumc.edu; Tel.: +1(614)-685-0657
}

\begin{abstract}
Direct cardiac reprogramming of fibroblasts into induced cardiomyocytes (iCMs) is a promising approach but remains a challenging technology of regenerative medicine for damaged myocardium. Efforts have been focused on improving the efficiency by understanding fundamental mechanisms. One of the major challenges is that the plasticity of cultured fibroblast varies batch to batch with unknown mechanisms. Here, we noticed that a portion of in vitro cultured fibroblasts have been activated to differentiate into myofibroblasts, marked by the expression of $\alpha S M A$, even in the primary cell culture of tissues. Both forskolin, which activates adenylyl cyclase and increases cAMP concentration, and TGF $\beta$ inhibitor SB431542 can efficiently suppress myofibroblast differentiation of cultured fibroblasts. However, SB431542 improved but forskolin blocked iCM reprogramming of fibroblasts that were infected with retroviruses of Gata4, Mef2c and Tbx5 (GMT). Moreover, inhibitors of cAMP downstream signaling pathways, PKA or CREB-CBP, significantly improved the efficiency of $\mathrm{iCM}$ reprogramming. Consistently, inhibition of p38/MAPK, another upstream regulator of CREB-CBP, also improved reprogramming efficiency. We then investigated if inhibition of these signaling pathways in primary cultured fibroblast could improve their plasticity for reprogramming, and found that preconditioning of cultured fibroblasts with CREB-CBP inhibitor significantly improved the cellular plasticity of fibroblasts to be reprogrammed, yielding $\sim 2$-fold amount of reprogrammed iCMs compared to that of untreated control cells. In conclusion, suppression of cAMP/PKA/CREB signaling axis improves fibroblast plasticity for direct cardiac reprogramming.
\end{abstract}

Keywords: induced cardiomyocyte; epigenetic reprogramming; heart regeneration; cell plasticity; transdifferentiation; CREB-CBP.

\section{Introduction}

Heart disease is the leading cause of global mortality. The most common form of heart disease is the ischemic heart disease, in which healthy myocardium falls short of oxygen and nutrient supplies and as a result undergoes apoptosis. Dead cardiomyocytes (CMs) are replaced by fibrotic scar tissue, that is generated by activated fibroblasts/myofibroblasts residing within the heart [1]. Adult mammalian cardiomyocytes have limited capacity to regenerate following an injury in heart tissue; therefore, ischemic heart requires an effective replacement therapy to recover heart function [2,3]. Recent studies found that mouse [4-7] and human fibroblasts [8-11] could be directly reprogrammed into functional induced-CMs (iCMs), which offers a potential therapeutic approach to prevent scar formation and replace dead myocardium.

Since the proof-of-concept discovery of direct cardiac reprogramming [4], many studies have focused on improving the reprogramming efficiency through investigating molecular and epigenetic mechanisms of reprogramming. Various strategies, including 
optimized gene delivery methods [12,13], inhibition of epigenetic barriers [14,15] and pro-fibrotic signaling [16-18], manipulation of cell-cycle in fibroblasts [14,19], and optimization of iCM cell culture [20,21], have been investigated to enhance the reprogramming efficiency. Majority of studies so far focused on reprogramming mechanisms after initiation of reprogramming; however, it has been known that the fibroblast state prior to reprogramming induction is also critical for a success of efficient reprogramming in vitro. Fibroblasts in the heart can be classified into four major cellular states: quiescent fibroblast, activated fibroblast, myofibroblast, and matrifibrocyte, each showing different plasticity with their special epigenetic inheritance [22,23]. A portion of fibroblasts showed resistance to epigenetic reprogramming [12,24] and varying degree of reprogramming had been also noticed in iCMs undergoing reprogramming [11,24,25], which might be due to the heterogeneous states of isolated fibroblasts. Pretreatments of in vitro cultured fibroblasts before initiation of reprogramming has significant impact on the reprogramming progress of fibroblasts. For example, Mitomycin-C treatment of permanently inactivating the proliferation of fibroblasts decreased the yield of reprogrammed iCM [15] while transiently cell-cycle synchronized fibroblasts have improved plasticity for reprogramming $[19,25]$. TGF $\beta$ is the most potent inducer of myofibroblasts differentiation [26]; it has been found that suppression of fibrotic TGF $\beta$ signaling pathway during the reprogramming significantly increased the efficiency of iCM reprogramming [27,28], suggesting that manipulation of fibroblast state, e.g. myofibroblast differentiation, plays a significant role in improving fibroblast plasticity for reprogramming. However, it has not been studied whether myofibroblast differentiation of in vitro cultured fibroblasts is associated with the plasticity of fibroblasts to be reprogrammed. Therefore, we decided to investigate the role of $\mathrm{CAMP} / \mathrm{PKA} / \mathrm{CREB}-\mathrm{CBP}$ signaling pathways in iCM reprogramming and explored the molecular mechanism that influences the fibroblast plasticity in epigenetic reprogramming in vitro.

In this study, we noticed that myofibroblast differentiation is generally activated in cultured fibroblasts, including neonatal mouse tail-tip fibroblasts (TTFs) and mouse embryonic fibroblasts (MEFs), which might be associated with the varying efficiency of iCM reprogramming. We studied the roles of TGF $\beta$ and cAMP signaling pathways in myofibroblast differentiation and $\mathrm{iCM}$ reprogramming of TTFs and MEFs. We next investigated the actions of chemical compounds, which inhibit p38/MAPK and the cAMP signaling downstream molecules (PKA and CREB-CBP), on iCM reprogramming and their role in regulation of the fibroblast plasticity, with a goal to better understand a signaling axis that promotes plasticity of mouse fibroblasts for reprogramming induction.

\section{Materials and Methods}

\section{Transgenic Mouse Line}

We used transgenic $\alpha$ MHC-GFP mice that were previously generated [4]. Animal experiments were approved by the Institutional Animal Care and Use Committee (IACUC) at Case Western Reserve University (2015-0058) and the Ohio State University (2019A00000085), and all animals handled according to the university guidelines.

\section{Neonatal Tail-tip Fibroblast Isolation}

Neonatal mouse tail-tip fibroblasts (TTFs) were prepared by explant-culture methods [29]. Briefly, tail-tips were harvested from neonatal $\alpha \mathrm{MHC}-\mathrm{GFP}$ transgenic mice (P0.5), and minced into small pieces $\left(<1 \mathrm{~mm}^{3}\right)$. The minced tissues were plated on gelatin-coated dishes for 5-7 days in fibroblast media (DMEM with 10\% FBS (Hyclone), NEAA and L-glutamine (Gibco)). Explanted TTFs were digested into single cells, filtered through $40 \mu \mathrm{m}$ cell strainer (Falcon, Fisher Scientific), and used for iCM reprogramming.

\section{Mouse Embryonic Fibroblast Isolation}

Mouse embryonic fibroblasts were isolated as published [19]. Briefly, MEFs were extracted from $\alpha$ MHC-GFP transgenic embryos (E13.5-14.5) in 0.125\% Trypsin/EDTA, 
filtered through $40 \mu \mathrm{m}$ cell strainer, and cultured in fibroblast media until the cultured cells became confluent. Cells were then either stored at $-80^{\circ} \mathrm{C}$ or freshly passaged for reprogramming.

For the pre-treatment experiments, TGF $\beta$ inhibitor SB431542 (1 $\mu \mathrm{mol} / \mathrm{L}, \mathrm{Cat} \# 13031$, Cayman Chem.), p38/MAPK inhibitor SB203580 (100 nmol/L, Cat\#1202, Tocris), or 2.5 $\mu \mathrm{mol} / \mathrm{L}$ CREB-CBP interaction inhibitor (Cat\#217505, Calbiochem) were added into the culture media $24 \mathrm{hrs}$ post-culture of freshly isolated MEFs. Cells were treated with compounds all the times, including culture and frozen media, until they are infected with GMT retroviruses for reprogramming.

\section{Direct Reprogramming and Flow Cytometry}

Retroviruses for reprogramming factors were generated as previously reported [4,19]. Briefly, pMX retroviral plasmids for individual transcription factors (GMT: Gata4, Mef2c, and Tbx5) were transfected into 90\% confluent Platinum E cells (Cell Biolabs) with FugeneHD (Promega) as suggested by manufacturer's protocol. Transfection media were removed next day and replaced with fresh PlatE media (DMEM with 10\% FBS). Approximately 48 hours after transfection, culture media containing viruses were harvested and filtered with $0.45 \mu \mathrm{M}$ filters (Nalgene, ThermoSci), and used freshly for iCM reprogramming.

Fibroblasts were seeded at a density of 120,000 cells per well in a 6-well plate one day before retrovirus infection, and were infected with a mixture of freshly-made GMT viruses $(0.5 \mathrm{ml}$ of each) with $8 \mu \mathrm{g} / \mathrm{ml}$ polybrene (Millipore) supplement. Next day, retroviral media was removed and replaced with fresh iCM media (DMEM/M199 (4:1) with $10 \%$ heat-inactivated FBS, NEAA, and L-glutamine) without or with chemical compounds; forskolin $(10 \mu \mathrm{mol} / \mathrm{L}, \mathrm{Cat}$ 6886, Sigma), TGF $\beta$ inhibitor SB431542 (1 $\mu \mathrm{mol} / \mathrm{L})$, p38/MAPK inhibitor SB203580 (100 nmol/L), CREB inhibitor (2.5 $\mu \mathrm{mol} / \mathrm{L})$, or PKA inhibitor H89 dihydrochloride ( $1 \mu \mathrm{mol} / \mathrm{L}$, Cat\# 2910, Tocris), or PKI 14-22 amide (peptide inhibitor of PKA; $5 \mu \mathrm{mol} / \mathrm{L}$, Cat\#2546, Tocris) for 7 days. Reprogrammed fibroblasts were maintained in iCM media with media change every 2-3 days. To evaluate the outcome of reprogramming, iCMs were digested into single cells with $0.05 \%$ Trypsin/EDTA and resuspended in FACS buffer (5\% FBS and 2 mM EDTA in 1X PBS). BD Accuri C6 flow cytometer (BD Biosciences) was used to evaluate percentage and absolute number of $\alpha \mathrm{MHC}-\mathrm{GFP}^{+} \mathrm{iCMs}$.

\section{Quantitative Real-time PCR (qRT-PCR)}

To evaluate gene expressions of cardiac markers in iCMs, reprogramming wells were harvested at Day7-8 post reprogramming and lysed in Trizol (Cat\#15596018, ThermoSci), and total RNA was extracted as per manufacturer's protocol. cDNA was generated from $2 \mu \mathrm{g}$ RNA samples using MultiScribe reverse transcription kit (Cat\#4311235, ThermoSci). Quantitative PCR assays were performed with SsoFast EvaGreen supermix (Cat\#1725201, BioRad) by a 7300 Real-Time PCR system (Applied Biosystems). The primers of cardiac markers are Myh6 (F: GCCCAGTACCTCCGAAAGTC; R: GCCTTAACATACTCCTCCTTGTC), Ryr2 (F: ACGGCGACCATCCACAAAG; R: AAAGTCTGTTGCCAAATCCTTCT), Tnnt2 (F: ACAGAGGAGGCCAACGTAGA; R: AAGTTGGGCATGAAGAGCCT), and Actc1 (F: TGCCATGTATGTCGCCATCC; R: CACCATCGCCAGAATCCAGA). The primers of myofibroblast markers include Acta2 (F: ATCACCAACTGGGACGACAT; R: CATACATGGCTGGGACATTG), Pten (F: TGGATTCGACTTAGACTTGACCT; R: GCGGTGTCATAATGTCTCTCAG), $\quad P x n$ (F: CAAACGGCCAGTGTTCTTGTC; R: TGTGTGGTTTCCAGTTGGGTA), and $V_{c l}$ (F: TGGACGGCAAAGCCATTCC; R: GCTGGTGGCATATCTCTCTTCAG). The expression of genes was quantified and normalized to a housekeeping gene Gapdh (F: AGGTCGGTGTGAACGGATTTG; R: TGTAGACCATGTAGTTGAGGTCA). Data was shown as a fold change of gene expression compared to GMT control group. 


\section{Assays of Myofibroblast Differentiation}

The expression of myofibroblast markers in cultured fibroblasts was evaluated by qRT-PCR and immunostaining. To suppress the differentiation of myofibroblasts, fibroblasts were treated with $1 \mu \mathrm{mol} / \mathrm{L} \mathrm{SB} 431542$ or $10 \mu \mathrm{mol} / \mathrm{L}$ forskolin for $48 \mathrm{hrs}$. Cultured cells were harvested and lysed in Trizol, and the mRNA expression of myofibroblast markers was evaluated by qRT-PCR as described above.

For immunostaining of a myofibroblast marker $\alpha$ SMA and a fibroblast marker Thy1, cultured fibroblasts were fixed with $4 \%$ PFA and incubated with primary antibodies of anti- $\alpha$ SMA (1:400, Cat.\# MA5-11547, ThermoFisher Scientific) and anti-Thy1 (1:400, Cat.\# 13-0903-81, ThermoFisher Scientific) at $4^{\circ} \mathrm{C}$ overnight, and then incubated with fluorescence conjugated secondary antibodies (Invitrogen). The stained samples were imaged by DMi8 Leica fluorescent microscope (Leica Microsystems).

\section{Statistical Analyses}

All data were expressed as mean \pm SEM. For statistical analysis, all experimental groups included at least three biological replicates, and the statistical significance was examined by two-way paired student's t-test. $P$ values of $<0.05$ were accepted as statistically significant. ${ }^{*} \mathrm{p}<0.05,{ }^{* *} \mathrm{p}<0.01,{ }^{* * *} \mathrm{p}<0.001 ; \# \mathrm{p}<0.05, \# \mathrm{p}<0.01, \# \# \mathrm{p}<0.001$.

\section{Results}

In vitro cultured fibroblasts contain differentiated myofibroblasts prior to the induction of reprogramming

Although a proper expression of reprogramming factors (e.g. GMT) in fibroblasts is essential to epigenetic reprogramming, the quality or status of fibroblasts prior to the induction of reprogramming is an important factor for successful and efficient reprogramming. We noticed that some batches of reprogrammed neonatal tail-fibroblast (TTF) had relatively good efficiency $(8.46 \pm 0.64, n=5$, Figure $1 \mathrm{~A}-\mathrm{B})$ while some had very poor efficiency or failed to be reprogrammed $(0.78 \pm 0.34, n=5$, Figure $1 C)$. Although many factors should be considered, we asked if it is associated with myofibroblast differentiation, which has been observed in in vitro cultured fibroblasts with increased expression of fibrotic genes [30,31]. Indeed, our immunostaining did show that myofibroblast marker, alpha smooth muscle actin ( $\alpha \mathrm{SMA}$ ) was expressed in many primary cultured TTFs (Figure 1D), and the expression of $\alpha S M A$ gene significantly increased in secondary-cultured cells $(2.72 \pm 0.58$ folds, $n=4, p=0.0116535)$ even only after one passage of primary cultured fibroblasts (Figure 1E). It has been reported in many laboratories that freshly-isolated fibroblasts, rather than passaged fibroblasts, are recommended to be used for a good efficiency of reprogramming [1,3,32]; therefore, we speculated that passaged fibroblasts loose plasticity for reprogramming via alternating fibroblast state, such as differentiating into myofibroblasts. 
A
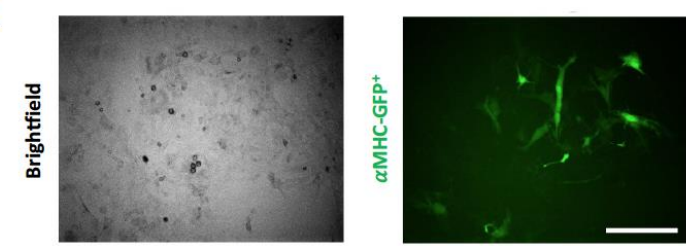

B
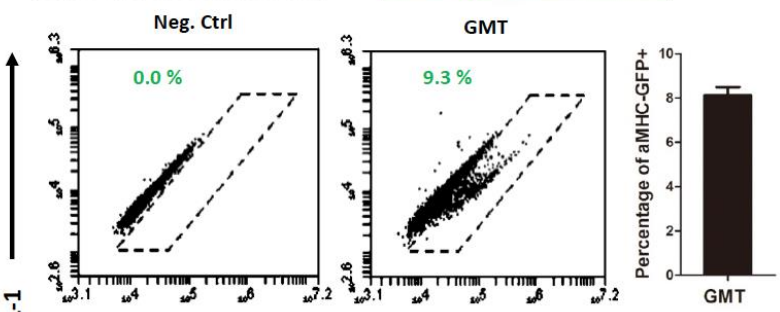

C
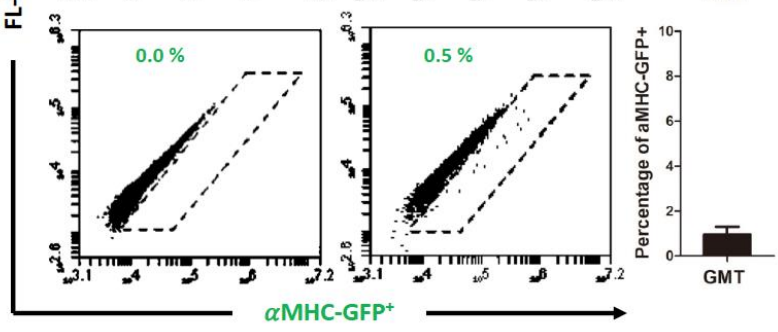

D
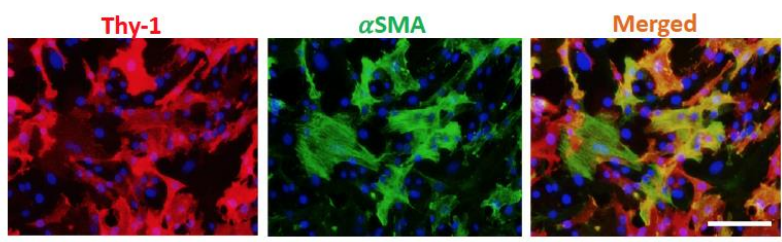

E

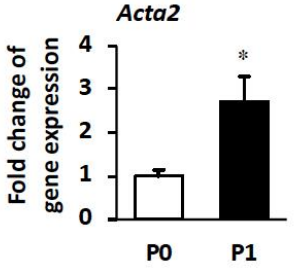

$\alpha-\mathrm{SMA}^{+}$

$\alpha-\mathrm{SMA}^{+}$

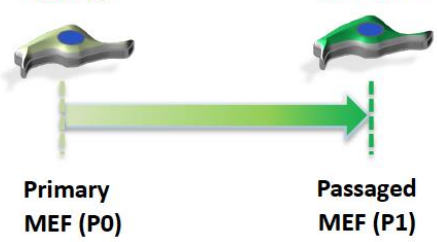

Figure 1. The plasticity of in vitro cultured fibroblasts varies. A) Brightfield (left) and GFP (right) representative images of iCM reprogramming (Day8) from tail-tip fibroblasts (TTFs). Bars indicate $50 \mu \mathrm{m}$. B-C) Representative FACS plots and average percentage of $\alpha M H C-\mathrm{GFP}^{+} \mathrm{iCMs}$ reprogrammed from TTFs with relatively good (B) and low (C) efficiency. D) Immunostaining of TTFs for fibroblast marker, Thy1, and myofibroblast marker, $\alpha \mathrm{SMA}$, showing that cultured fibroblasts were differentiated into myofibroblasts. Bars indicate $200 \mu \mathrm{m}$. E) qRT-PCR shows that gene expression of Acta2, a myofibroblast marker, was significantly higher in single-passaged cells (P1) than that in primary fibroblasts (P0), indicating myofibroblast differentiation in cultured fibroblasts. ${ }^{*} p<0.05$ vs. P0 fibroblasts.

To prevent myofibroblast differentiation of cultured cells, we treated fresly-isolated TTFs with a TGF $\beta$ inhibitor (SB431542) or a cAMP activator (forskolin) (Figure 2A) for 48 hours. We found that both compounds affectively suppressed myofibroblast differentiation, indicated by the significantly-decreased expression of $\alpha S M A$ in Thy $1^{+}$ fibroblast population (Figure 2B). Our qRT-PCR confirmed that the expression of myofibroblast late-differentiation marker, $\alpha$ SMA, was significantly suppressed by both SB431542 and forskolin (Figure 2C). Interestingly, SB431524 increased the expression of myofibroblast early-differentiation markers, including Pten (tensin), Pxn (paxillin), and Vcl (vinculin); while forskolin suppressed the expression of $\mathrm{Vcl}$ (Figure 2D). These results suggested that myofibroblasts differentiation of cultured fibroblasts can be efficiently suppressed by inhibiting TGF $\beta$ signaling or activating cAMP signaling; however, inhibition of TGF $\beta$ signaling pathway possibly targets the late stage of myofibroblast differentiation, while the early stage is regulated by activation cAMP signaling pathway. 


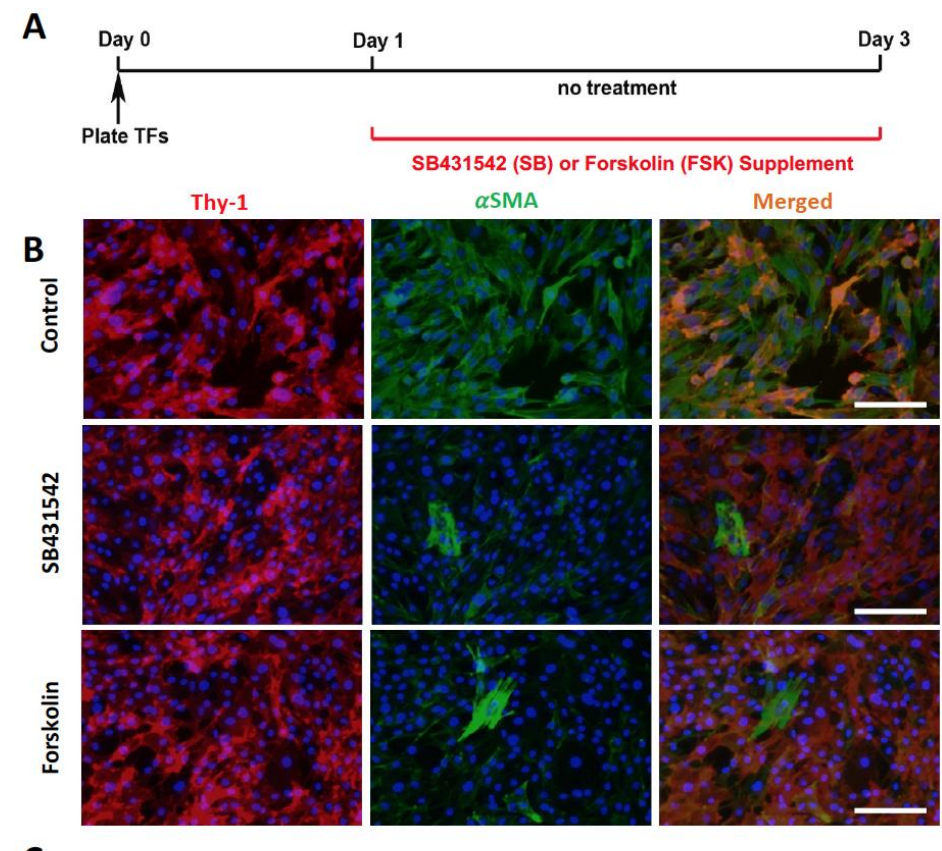

C
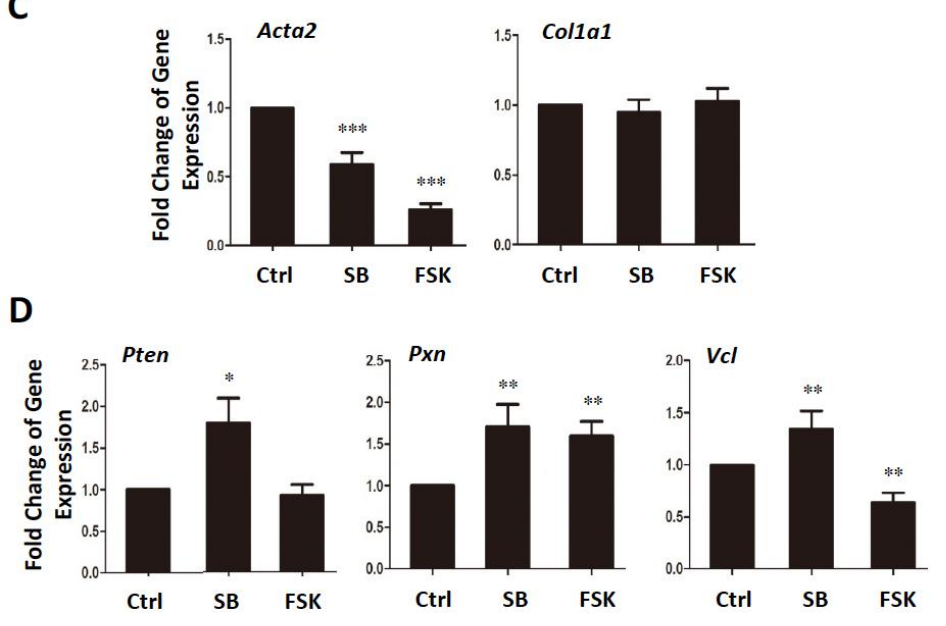

Figure 2. Inhibition of TGF $\beta$ or activation of cAMP signaling pathways suppress myofibroblast differentiation of cultured fibroblasts. A) Experimental design of compound treatments. B) Immunostaining of Thy1 (a fibroblast marker) and aSMA (a myofibroblast marker) shows that the myofibroblast differentiation was inhibited by TGF $\beta$ inhibitor SB431542 (SB) or forskolin (FSK). Bars indicate $100 \mu \mathrm{m}$. C-D) Fold changes of the mRNA expression of myofibroblast differentiation markers were significantly decreased in SB- or FSK-treated TTFs compared to untreated control (Ctrl) cells. ${ }^{*} \mathrm{p}<0.05,{ }^{* *} \mathrm{p}<0.01,{ }^{* * *} \mathrm{p}<0.001$ vs. Ctrl.

Post-induction treatments of SB431542 and Forskolin have opposite effects on iCM reprogramming

Next, we treated reprogrammed TTFs with SB431542 or forskolin for 7 days after infection of GMT retroviruses (Figure 3A) and studied the efficiency of reprogrammed $\alpha \mathrm{MCH}-\mathrm{GFP}^{+} \mathrm{iCM}$. Consistent with previous reports $[17,18]$, inhibition of TGF $\beta$ signaling by SB431542 significantly improved the reprogramming efficiency $(1.80 \pm 0.20$ folds, $n=4$, $\mathrm{p}=0.029$ ) of TTFs (Figure 3B-D). Surprisingly, activation of cAMP signaling by forskolin dramatically decreased the yield of reprogrammed $\alpha \mathrm{MCH}-\mathrm{GFP}^{+}$cells $(0.31 \pm 0.17$ folds, $\mathrm{n}=4, \mathrm{p}=0.012$ ) from TTFs (Figure 3B-D). The activation of cardiac muscle genes by GMT, including Myh6, Ryr2, Tnnt2 and Actc1, were significantly inhibited by forskolin, while SB431542 facilitated their activations (Figure 3E). The inhibition of iCM reprogramming by forskolin was also observed in mouse embryonic fibroblasts (MEFs). Activation of cAMP signaling by forskolin significantly suppress iCM reprogramming of MEFs and 
yielded much less $\alpha \mathrm{MCH}-\mathrm{GFP}^{+} \mathrm{iCMs}$ (Figure 4). Our results suggested that activation of cAMP signaling pathway might be a barrier in the epigenetic reprogramming of iCMs.

A

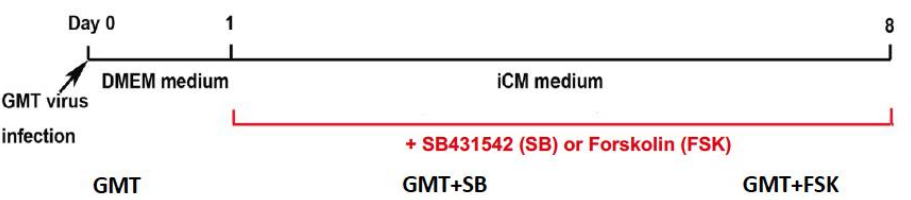

B
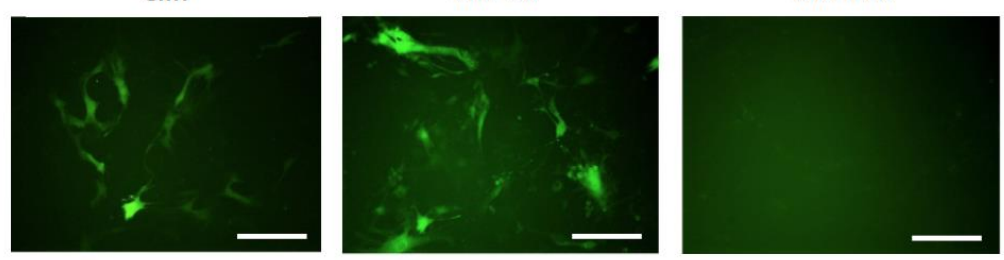

C

Neg. Ctrl
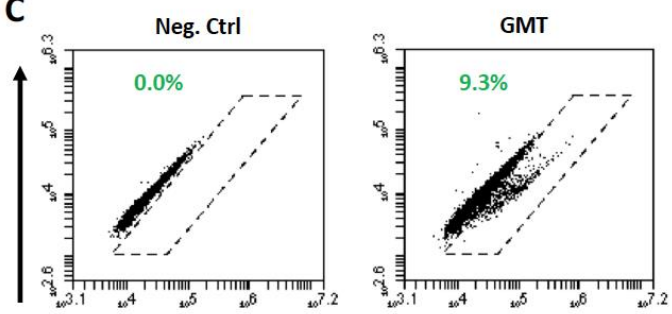

E
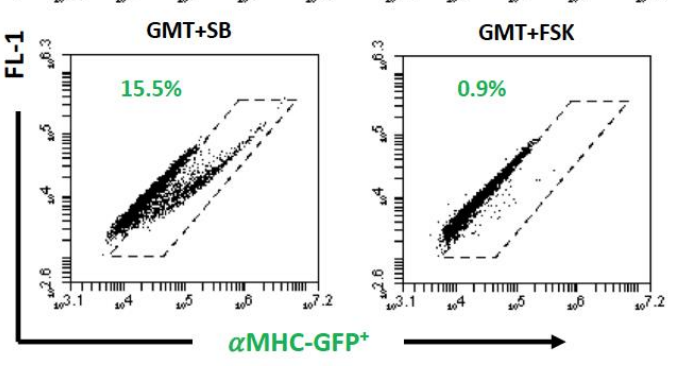

D
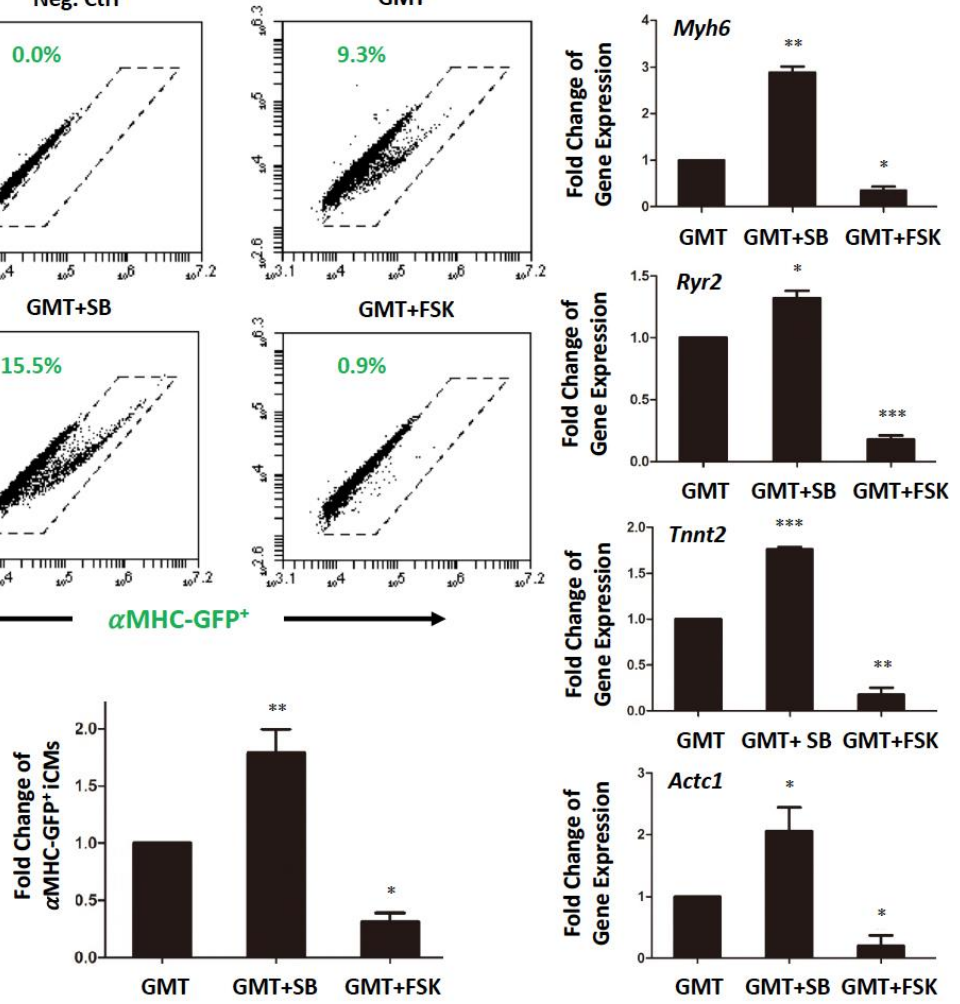

Figure 3. Post-induction treatments of SB431542 (SB) and forskolin (FSK) have opposite effect on iCM reprogramming of tail-tip fibroblasts (TTF). A) Experimental strategy of post-induction treatment during iCM reprogramming. B-C) Representative fluorescent images (B) and FACS plots (C) of iCM reprogramming at Day8 after infection. Bars indicate $50 \mu \mathrm{m}$. D) Average fold changes of reprogrammed $\alpha \mathrm{MCH}-\mathrm{GFP}^{+} \mathrm{iCMs}$ by SB431542 and FSK. E) Fold changes of cardiac gene expression in reprogrammed fibroblasts, including Myh6, Ryr2, Tnnt2, and Actc1. ${ }^{*} \mathrm{p}<0.05$, ${ }^{* *} \mathrm{p}<0.01$, or *** $\mathrm{p}<0.001$ vs. GMT ctrl. 
A

A GMT
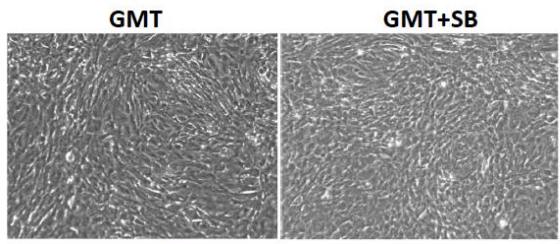

GMT+FSK
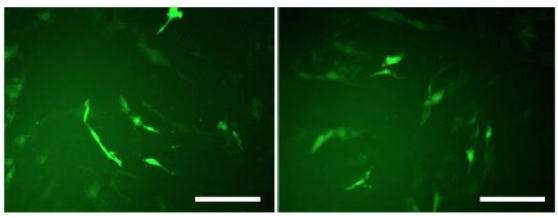

B
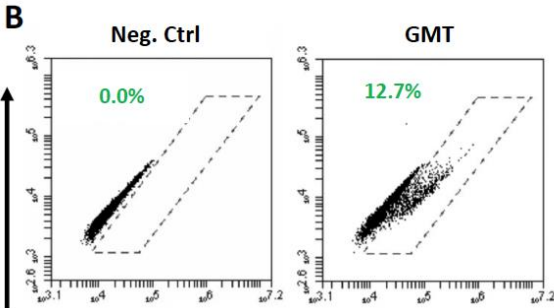

C
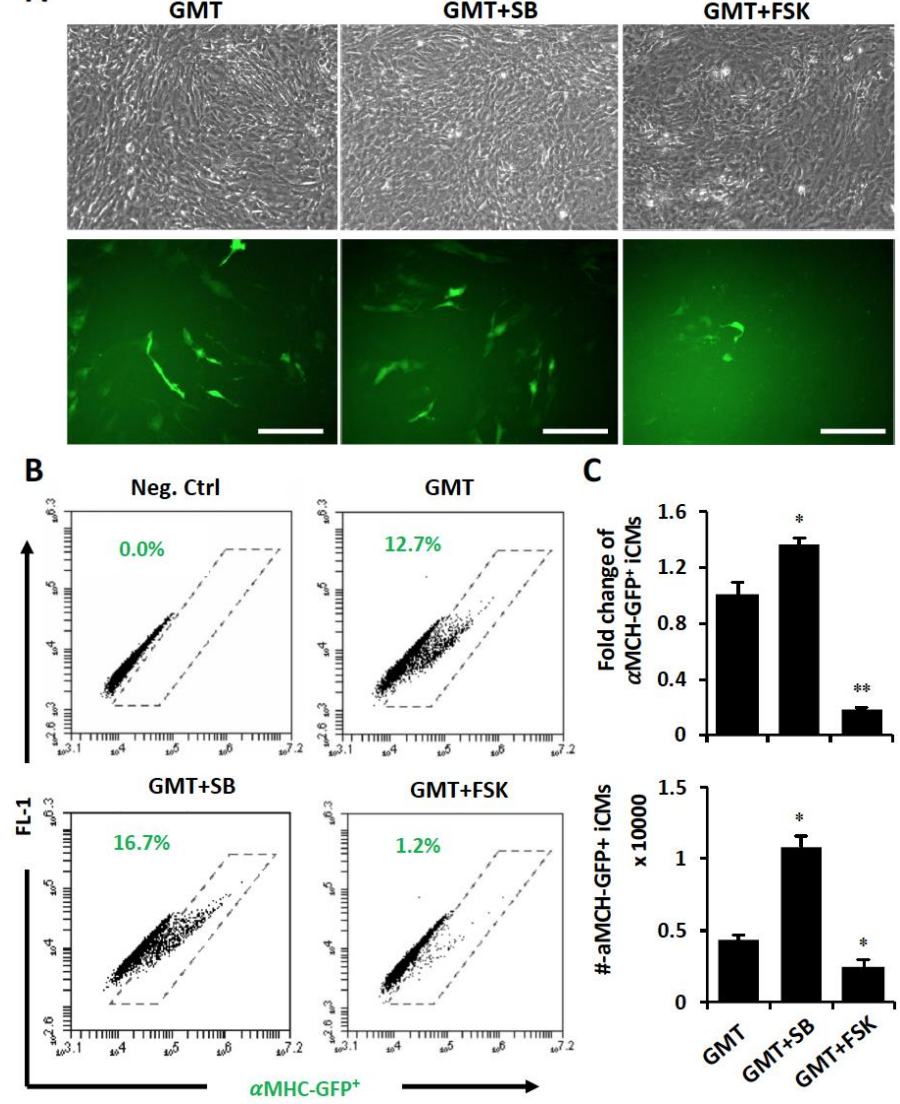

Figure 4. Forskolin inhibits iCM reprogramming of MEFs. A-B) Representative brightfield (upper lane) and fluorescent (lower lane) images (A) and FACS plots (B) of $\alpha \mathrm{MCH}-\mathrm{GFP}^{+} \mathrm{iCMs}$ at Day 8 of reprogramming with post-induction treatments of SB431542 (SB) or forskolin (FSK). Bars indicate $50 \mu \mathrm{m}$. C) Average fold changes of GMT-reprogrammed $\alpha \mathrm{MCH}-\mathrm{GFP}^{+} \mathrm{iCMs}$ by SB $(1.37 \pm 0.04 ; \mathrm{n}=3)$ or FSK $(0.17 \pm 0.02 ; n=3)$. Average yield of $\alpha M C H-G F P+i C M s$ by GMT $(4,302 \pm 377$ cells; $n=3)$ was increased to $10,851 \pm 759$ cells $(n=3)$ by SB but decreased to $2,452 \pm 507$ cells $(n=3)$ by FSK. ${ }^{*} p<0.05$ or ** $\mathrm{p}<0.01$ vs. GMT ctrl.

\section{Inhibition of cAMP donstream PKA signaling post-GMT induction improves reprogramming}

Forskolin activates critical signaling pathways via a secondary messenger, cAMP [33,34] (Figure 5A), which activates protein kinase A (PKA) [35] and results in phosphorylation of numerous targets, including transcriptional regulators, e.g. CREB [36], that mediate critical biological processes. Therefore, we asked if PKA signaling pathway mediates the observed inhibition of iCM reprogramming by forskolin-induced cAMP. We studied a post-induction treatment of PKI 14-22 amide, a specific peptide that binds on catalytic site of PKA and inhibits its kinase activity, and found that PKI significantly improved the reprogramming efficiency ( $1.25 \pm 0.08$ folds, $n=6, p=0.0098$; Figure $5 B-C)$ and increased the number of reprogrammed $\alpha \mathrm{MCH}-\mathrm{GFP}^{+}$iCMs $(18,352 \pm 7123$ vs. $12707 \pm 4741$ of the GMT control group; $n=8, p=0.0416$; Figure $5 \mathrm{D}$ ). The enhancement of $\mathrm{iCM}$ reprogramming by PKI was also observed in TTFs (data no shown). We had also applied H89 dihydrochloride, a chemical inhibitor of PKA, post-GMT induction and observed a consistent enhancement of reprogramming with increased percentage $(1.27 \pm 0.17, n=7$, $\mathrm{p}=0.0279)$ and absolute number $(14,989 \pm 6588$ vs. $11,062 \pm 5588$ of the GMT control group; $\mathrm{n}=7, \mathrm{p}=0.0276$ ) of reprogrammed $\alpha \mathrm{MCH}-\mathrm{GFP}^{+}$iCMs (Figure 5E).

We next investigated CREB, a downstream target of PKA signaling pathway, and p38/MAPK pathway, which is known to interact with both PKA and CREB pathways $[36,37]$. We found that a chemical inhibitor of p38/MAPK signaling (p38i), SB203580, could significantly enhance the efficiency (1.46 \pm 0.05 folds; $n=7, p=0.0001)$ of iCM 
reprogramming and increased the number of reprogrammed $\alpha \mathrm{MCH}-\mathrm{GFP}^{+} \mathrm{iCMs}$ from $24,044 \pm 6578$ cells in the GMT control group to $39,062 \pm 10,401$ ( $n=7, p=0.0087$ ) (Figure 5F-G). Similarly, an inhibitor of CREB and CBP interaction improved the efficiency of reprogramming $(1.79 \pm 0.08$ folds; $n=7, p<0.001)$ and increased cell number $(46,790 \pm 11,584$; $\mathrm{n}=7, \mathrm{p}=0.005)$ of $\mathrm{iCM}$ compared to GTM ctrl. Noticeably, CREBi was more effective than p38i at improving both efficiency $(p=0.0039)$ and cell number $(p=0.0122)$ of iCMs (Figure $5 \mathrm{G})$. The enhancement of reprogramming by CREBi was also observed in cardiac fibroblasts (Data not shown). Our results demonstrate that CREB, a major downstream signaling of both PKA and p38 signaling pathways, plays an imporatant role in regulation of $\mathrm{iCM}$ reprogramming.
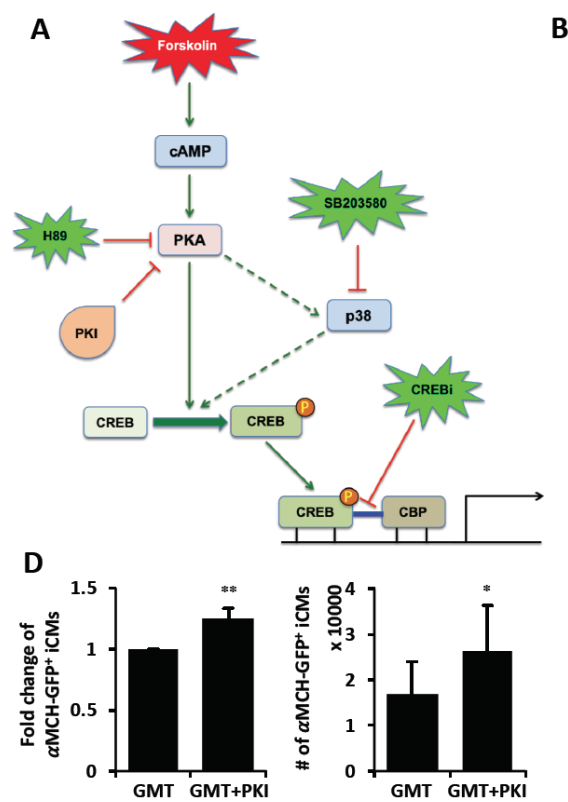

$\mathbf{E}$
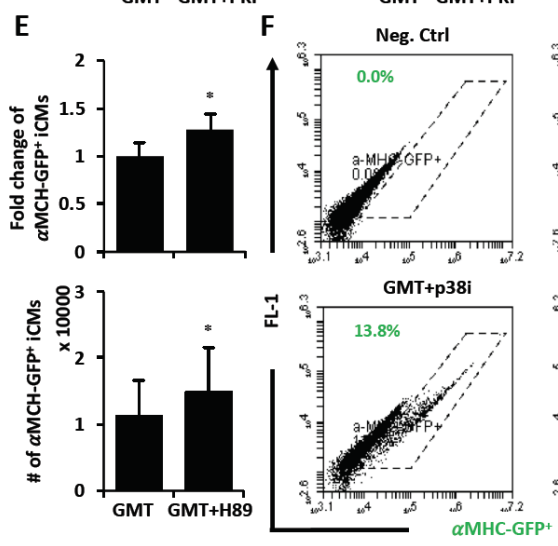

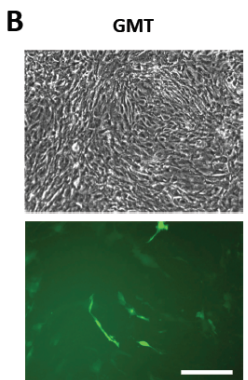

GMT+PKI
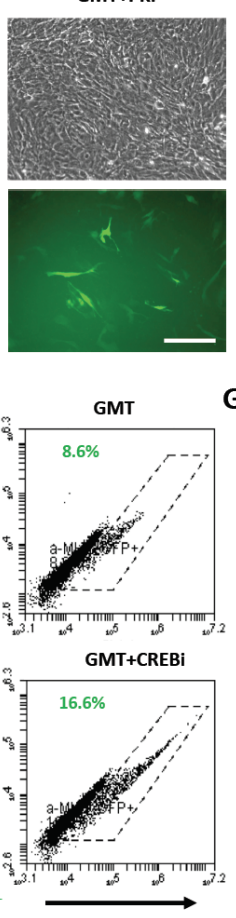
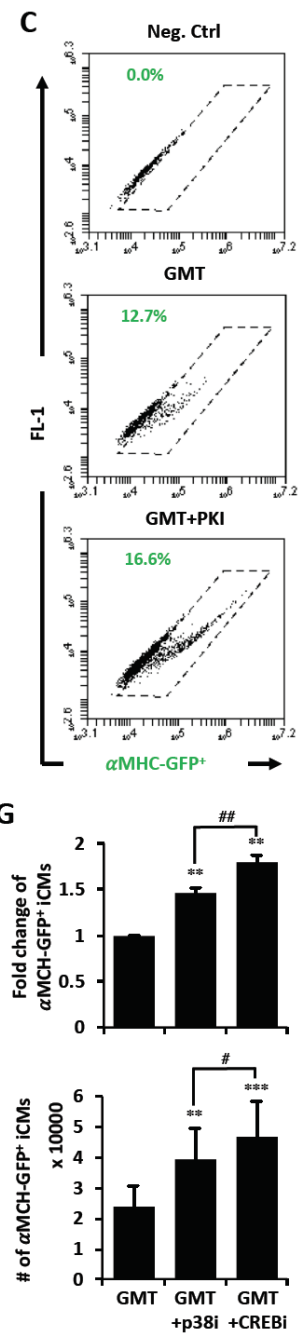

Figure 5. Inhibition of PKA signaling pathway improves iCM reprogramming of MEFs. A) Diagram showing cAMP/PKA/CREB signaling pathway activated by forskolin. B-C) Representative brightfield and GFP images (B) and FACS plots (C) of $\alpha M C H-G F P^{+}$iCMs treated with or w/o a PKA peptide inhibitor (PKI). Bars indicate $50 \mu \mathrm{m}$. D) Bar graphs show fold change of percentage (left panel) and absolute number (right panel) of $\alpha \mathrm{MCH}-\mathrm{GFP}^{+} \mathrm{iCMs}$ in reprogramming of GMT or GMT+PKI. E) H89 dichloride, a chemical inhibitor of Protein kinase A, increased the percentage

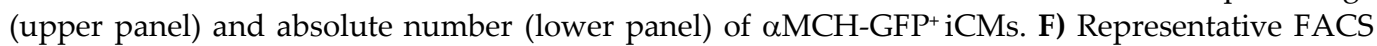
plots of GMT reprogramming without or with a chemical inhibitor of p38 (p38i) SB203580 or a CREB inhibitor (CREBi). G) Average fold changes of the percentage (upper panel) and the absolute number (lower panel) of reprogrammed $\alpha M C H-G F P^{+} \mathrm{iCMs}$ by $\mathrm{p} 38 \mathrm{i}$ or CREBi. ${ }^{*} \mathrm{p}<0.05,{ }^{* *} \mathrm{p}<0.01$, *** $\mathrm{p}<0.001$ vs. GMT ctrl; \#p<0.05, \#\#p<0.01 vs. GMT+p38i.

Pretreatment of primary fibroblasts with CREB-CBP inhibitor improves fibroblast plasticity for reprogramming 
We further asked whether any of these signaling pathways could maintain the plasticity of in vitro cultured fibroblasts to be reprogrammed. We treated fibroblasts with TGF $\beta$ inhibitor (TGF $\beta \mathrm{i}$ ), $\mathrm{p} 38 \mathrm{i}$, or CREBi from the initial time of primary cultures until MEFs were used for GMT-induction (Figure 6A). We didn't notice obvious morphology changes of primary cultured cells among groups with or without chemical inhibitors. Primary culture of MEFs were all proliferating healthily with high cell density in all groups (Figure 6B). We found that chemical inhibitors did have different effect on the proliferation capability of MEFs after freezing and passing of secondary cell culture; $\mathrm{p} 38 \mathrm{i}$ and CREBi improved cell proliferation with much higher density of MEFs than the control group, while TGF $\beta \mathrm{i}$ suppressed the proliferation of MEFs (Figure 6C). Remarkably, CREBi-pretreated MEFs showed a significantly-enhanced plasticity to be reprogrammed by GMT, with a better reprogramming efficiency $(2.02 \pm 0.58$ folds; $n=3$, $\mathrm{p}=0.0004)$ and an increased number of reprogrammed $\alpha \mathrm{MCH}-\mathrm{GFP}^{+} \mathrm{iCMs}(71,094 \pm 9311$ cells vs. $28,463 \pm 4963$ cells in the untreated control group; $n=3, p=0.0160$ ), while pretreatments of TGF $\beta i$ or $\mathrm{p} 38 \mathrm{i}$ had no significant effect on the reprogramming plasticity. Our study demonstrated that inhibition of CREB signaling improves the plasticity of fibroblasts for cardiac epigenetic reprogramming.

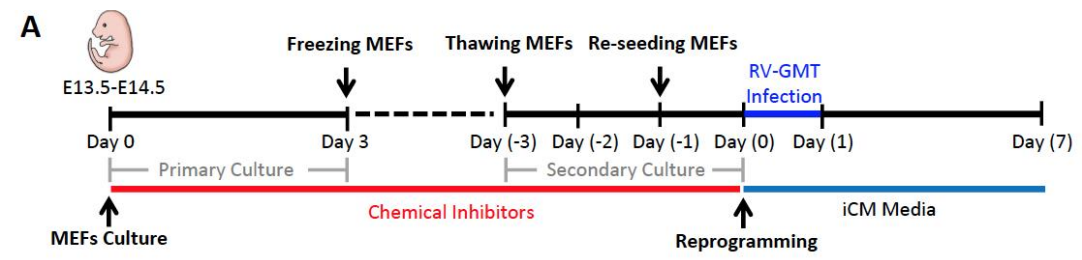

B Day 3 of Primary Culture
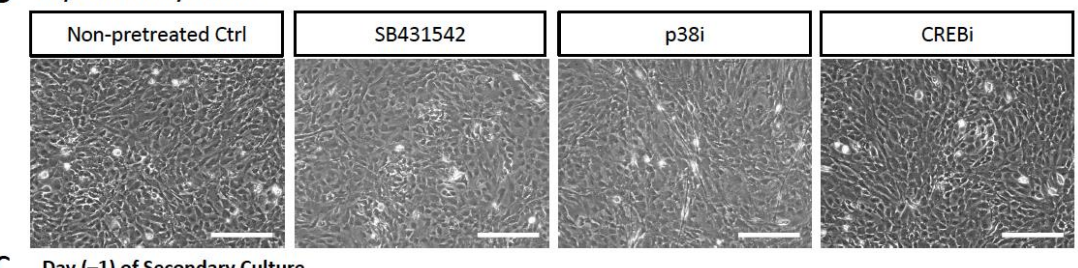

C Day (-1) of Secondary Cultur
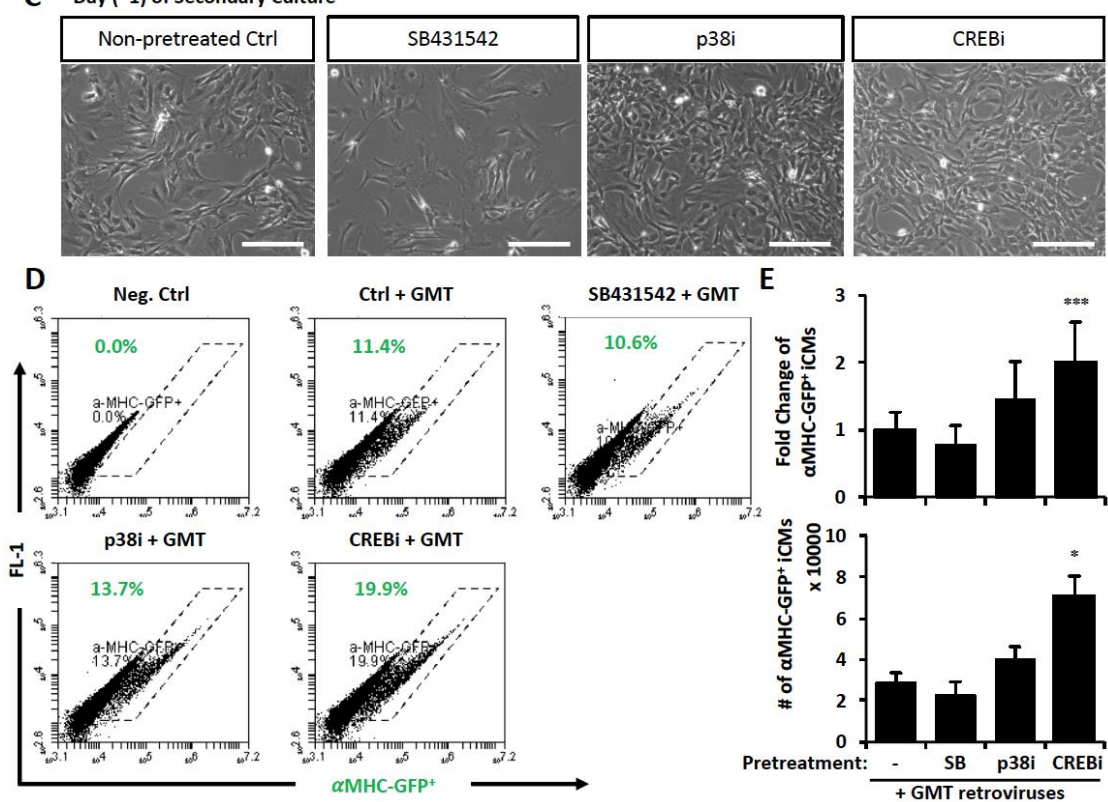

Figure 6. Pretreatment of MEFs with CREB-CBP inhibitor preserves cellular plasticity for reprogramming. A) Experimental design of MEF culture and pretreatments of TGF $\beta$ inhibitor (SB431542), p38 inhibitor (p38i), or CREB inhibitor (CREBi) before direct cardiac reprogramming by GMT retrovirus. B) Representative images of compound-treated MEFs at Day 3 of primary culture. C) Representative images of MEFs at Day (-1) of secondary culture. D) Representative FACS plots of $\alpha \mathrm{MCH}-\mathrm{GFP}^{+} \mathrm{iCMs}$ reprogrammed from MEFs without or with pretreatments of chemical com- 
pounds. E) Average fold changes of the percentage (upper panel) and absolute number (lower panel) of $\alpha \mathrm{MCH}-\mathrm{GFP}^{+} \mathrm{iCMs}$ from MEFs pretreated with SB431542, p38i or CREBi. MEFs without pre-treatment of the same-batch culture were used as the ctrl group. ${ }^{*} \mathrm{p}<0.05,{ }^{* * *} \mathrm{p}<0.0005$ vs. non-pretreated ctrl. Bars indicate $50 \mu \mathrm{m}$.

\section{Discussion}

In this study, we investigated novel signaling pathways, including TGF $\beta$, cAMP/PKA, and p38, that are involved in direct cardiac reprogramming and found that inhibition of $\mathrm{CAMP} / \mathrm{PKA} / \mathrm{CREB}$ signaling axis could preserve the plasticity of cultured fibroblast to be reprogrammed.

In vitro cultured fibroblasts have different cellular states that affect the efficiency of epigenetic reprogramming, yielding a varied efficiency from batch to batch. TGF $\beta$ inhibitor SB431542 has been reported to significantly enhance iCM reprogramming $[16,17,27]$. Myofibroblast differentiation commonly exists in in vitro cultured fibroblasts with increased expression of fibrotic genes [30,31]; and inhibition of TGF $\beta$ signaling can effectively suppress myofibroblast differentiation [38,39]. These discoveries suggest that myofibroblast status may be an epigenetic barrier for direct cardiac reprogramming and suppression of myofibroblast differentiation might be a good strategy to improve the reprogramming efficiency. In our study, cAMP signaling pathway, activated by forskolin, could suppress myofibroblast differentiation, as previously reported [40,41]. It sounds a rational hypothesis that forskolin may enhance reprogramming efficiency, particularly when exclusion of forskolin from the chemical cocktail of reprogramming factors significantly decreased induction of iCMs [42]. However, in our study, forskolin blocked reprogramming induced by GMT transcription-factor cocktail, which suggests that iCM reprogramming induced by chemical or transcription-factor cocktails may differentially require cAMP signaling. Although both TGF $\beta$ inhibitor and cAMP signaling activation suppress myofibroblast differentiation, we did observe the difference that SB431542 promoted early stage of differentiation, indicated by increased expression of early markers, but suppressed late stage of differentiation of myofibroblasts, indicated by decreased expression of $\alpha \mathrm{SMA}$, while forskolin could stage-independently suppress myofibroblast differentiation, which might be the underlying mechanism of how two compounds produce opposite effects on GMT-induced reprogramming of fibroblasts.

As one type of second messengers, cAMP has a broad range of targets in various signaling pathways, among which PKA signaling is a well-known cAMP target. Previously, it has been reported that inhibition of PKA signaling pathway improves reprogramming of iPSCs [43]. In this study, inhibition of PKA by a chemical compound (H89) or specific peptide (PKI 14-22 amide) also increased the yield of iCM reprogramming. We next studied one of the well-known downstream targets of PKA signaling, CREB, which regulates fibroblast [44-49] and cardiac function and disease [50-52]. We found that CREB inhibitor improves iCM reprogramming and inhibition of p38 signaling, which also regulate the phosphorylation of CREB, consistently enhanced reprogramming efficiency. These discoveries demonstrate that, post-initiation of GMT-mediated reprogramming, inhibition of cAMP/PKA/CREB axis alters epigenetic status and enhances direct cardiac reprogramming.

We asked if these signaling pathways, which suppress myofibroblast differentiation, could modify or preserve cultured fibroblasts in a more plastic state to be reprogrammed. We pretreated primary culture of fibroblasts with different inhibitors before the induction of reprogramming and investigated their plasticity to be reprogrammed into $\alpha \mathrm{MCH}-\mathrm{GFP}^{+} \mathrm{iCMs}$ by GMT. Neither TGF $\beta$ inhibitor nor p38 inhibitor improved the reprogramming efficiency of secondary-culture fibroblasts; although inhibitors of both signaling pathways have been reported to suppress myofibroblast differentiation $[38,53,54]$. Interestingly, CREBi-treated fibroblasts have better plasticity and more of them were reprogrammed into $\alpha \mathrm{MCH}-\mathrm{GFP}^{+} \mathrm{iCMs}$ than untreated control fibroblasts. The mechanism of preserved plasticity via CREB inhibition is beyond the suppression of myofibroblast differentiation. Cell-cycle synchronization facilitates the progression of 
iCM reprogramming [19], and inhibition of CREB signaling could suppress cell cycle in some cell lines [55-57]. In our study, however, we didn't observe that CREB inhibitor significantly change the cell-cycle phases of cultured MEFs (data not shown), therefore its mechanism in reprogramming is possibly not related to cell cycle. More investigations are needed to understand the mechanism of how CREB regulates plasticity of fibroblasts and how it benefits to reprogramming. Nevertheless, our discovery of preserving the reprogramming plasticity of cultured fibroblasts by inhibiting cAMP/PKA/CREB axis provides a better mechanistic understanding of direct cardiac reprogramming in vitro.

Author Contributions: Conceptualization, E.B. and J.D.F.; methodology, E.B., Y.S., A.T.D., and D.Y.; validation, E.B. and J.D.F; formal analysis, E.B., Y.S. and P.S.; investigation, E.B., Y.S. A.T.D., P.S., and D.Y.; resources, J.D.F.; data curation, E.B., Y.S., A.T.D., and P.S.; writing-original draft preparation, E.B.; writing - review and editing, JD.F.; visualization, E.B.; supervision, J.D.F.; project administration, J.D.F.; funding acquisition, J.D.F. All authors have read and agreed to the published version of the manuscript.

Funding: This research was funded by the Start-up Fund from The Ohio State University (to J.D.F). J.D.F. was also supported by American Heart Association (grant number 13SDG14580035) and NIH (grant number 1R01HL139006).

Institutional Review Board Statement: The study was conducted according to the university guidelines. All animal experiments were approved by the Institutional Animal Care and Use Committee (IACUC) at Case Western Reserve University (2015-0058) and the Ohio State University (2019A00000085).

Conflicts of Interest: The authors declare no conflict of interest. The funders had no role in the design of the study; in the collection, analyses, or interpretation of data; in the writing of the manuscript, or in the decision to publish the results.

\section{References}

1. Bektik, E.; Fu, J.D. Ameliorating the Fibrotic Remodeling of the Heart through Direct Cardiac Reprogramming. Cells 2019, 8, doi:10.3390/cells8070679.

2. van Berlo, J.H.; Molkentin, J.D. An emerging consensus on cardiac regeneration. Nat Med 2014, 20, 1386-1393, doi: $10.1038 / \mathrm{nm} .3764$.

3. Fu, J.D.; Srivastava, D. Direct reprogramming of fibroblasts into cardiomyocytes for cardiac regenerative medicine. Circ J 2015, 79, 245-254, doi:10.1253/circj.CJ-14-1372.

4. Ieda, M.; Fu, J.D.; Delgado-Olguin, P.; Vedantham, V.; Hayashi, Y.; Bruneau, B.G.; Srivastava, D. Direct Reprogramming of Fibroblasts into Functional Cardiomyocytes by Defined Factors. Cell 2010, 142, 375-386, doi:10.1016/j.cell.2010.07.002.

5. Jayawardena, T.M.; Egemnazarov, B.; Finch, E.A.; Zhang, L.; Payne, J.A.; Pandya, K.; Zhang, Z.; Rosenberg, P.; Mirotsou, M.; Dzau, V.J. MicroRNA-mediated in vitro and in vivo direct reprogramming of cardiac fibroblasts to cardiomyocytes. Circ Res 2012, 110, 1465-1473, doi:10.1161/CIRCRESAHA.112.269035.

6. Qian, L.; Huang, Y.; Spencer, C.I.; Foley, A.; Vedantham, V.; Liu, L.; Conway, S.J.; Fu, J.-d.D.; Srivastava, D. In vivo reprogramming of murine cardiac fibroblasts into induced cardiomyocytes. Nature 2012, 485, 593-598, doi:10.1038/nature11044.

7. Song, K.; Nam, Y.J.; Luo, X.; Qi, X.; Tan, W.; Huang, G.N.; Acharya, A.; Smith, C.L.; Tallquist, M.D.; Neilson, E.G., et al. Heart repair by reprogramming non-myocytes with cardiac transcription factors. Nature 2012, 485, 599-604, doi:10.1038/nature11139.

8. Fu, J.-D.D.; Stone, N.R.; Liu, L.; Spencer, C.I.; Qian, L.; Hayashi, Y.; Delgado-Olguin, P.; Ding, S.; Bruneau, B.G.; Srivastava, D. Direct reprogramming of human fibroblasts toward a cardiomyocyte-like state. Stem cell reports 2013, 1, 235-247, doi:10.1016/j.stemcr.2013.07.005. 
9. Nam, Y.J.; Song, K.; Luo, X.; Daniel, E.; Lambeth, K.; West, K.; Hill, J.A.; DiMaio, J.M.; Baker, L.A.; Bassel-Duby, R., et al. Reprogramming of human fibroblasts toward a cardiac fate. Proc Natl Acad Sci U S A 2013, 110, 5588-5593, doi:10.1073/pnas.1301019110.

10. Wada, R.; Muraoka, N.; Inagawa, K.; Yamakawa, H.; Miyamoto, K.; Sadahiro, T.; Umei, T.; Kaneda, R.; Suzuki, T.; Kamiya, K., et al. Induction of human cardiomyocyte-like cells from fibroblasts by defined factors. Proc Natl Acad Sci U S A 2013, 110, 12667-12672, doi:10.1073/pnas.1304053110.

11. Bektik, E.; Dennis, A.; Prasanna, P.; Madabhushi, A.; Fu, J.-D.D. Single cell qPCR reveals that additional HAND2 and microRNA-1 facilitate the early reprogramming progress of seven-factor-induced human myocytes. PloS one 2017, 12, doi:10.1371/journal.pone.0183000.

12. Wang, L.; Liu, Z.; Yin, C.; Asfour, H.; Chen, O.; Li, Y.; Bursac, N.; Liu, J.; Qian, L. Stoichiometry of Gata4, Mef2c, and Tbx5 Influences the Efficiency and Quality of Induced Cardiac Myocyte Reprogramming. Circulation Research 2015, 116, 237-244, doi:10.1161/CIRCRESAHA.116.305547.

13. Miyamoto, K.; Akiyama, M.; Tamura, F.; Isomi, M.; Yamakawa, H.; Sadahiro, T.; Muraoka, N.; Kojima, H.; Haginiwa, S.; Kurotsu, S., et al. Direct In Vivo Reprogramming with Sendai Virus Vectors Improves Cardiac Function after Myocardial Infarction. Cell stem cell 2018, 22, 91-10300000, doi:10.1016/j.stem.2017.11.010.

14. Zhou, Y.; Wang, L.; Liu, Z.; Alimohamadi, S.; Yin, C.; Liu, J.; Qian, L. Comparative Gene Expression Analyses Reveal Distinct Molecular Signatures between Differentially Reprogrammed Cardiomyocytes. Cell Reports 2017, 20, 3014-3024, doi:10.1016/j.celrep.2017.09.005.

15. Zhou, Y.; Wang, L.; Vaseghi, H.R.; Liu, Z.; Lu, R.; Alimohamadi, S.; Yin, C.; Fu, J.-D.D.; Wang, G.G.; Liu, J., et al. Bmi1 Is a Key Epigenetic Barrier to Direct Cardiac Reprogramming. Cell stem cell 2016, 18, 382-395, doi:10.1016/j.stem.2016.02.003.

16. Muraoka, N.; Yamakawa, H.; Miyamoto, K.; Sadahiro, T.; Umei, T.; Isomi, M.; Nakashima, H.; Akiyama, M.; Wada, R.; Inagawa, K., et al. MiR-133 promotes cardiac reprogramming by directly repressing Snai1 and silencing fibroblast signatures. The EMBO journal 2014, 33, 1565-1581, doi:10.15252/embj.201387605.

17. Ifkovits, J.L.; Addis, R.C.; Epstein, J.A.; Gearhart, J.D. Inhibition of TGFbeta signaling increases direct conversion of fibroblasts to induced cardiomyocytes. PLoS One 2014, 9, e89678, doi:10.1371/journal.pone.0089678.

18. Zhao, Y.; Londono, P.; Cao, Y.; Sharpe, E.J.; Proenza, C.; O'Rourke, R.; Jones, K.L.; Jeong, M.Y.; Walker, L.A.; Buttrick, P.M., et al. High-efficiency reprogramming of fibroblasts into cardiomyocytes requires suppression of pro-fibrotic signalling. Nature communications 2015, 6, 8243, doi:10.1038/ncomms9243.

19. Bektik, E.; Dennis, A.; Pawlowski, G.; Zhou, C.; Maleski, D.; Takahashi, S.; Laurita, K.R.; Deschenes, I.; Fu, J.D. S-phase Synchronization Facilitates the Early Progression of Induced-Cardiomyocyte Reprogramming through Enhanced Cell-Cycle Exit. Int J Mol Sci 2018, 19, doi:10.3390/ijms19051364.

20. Yamakawa, H.; Muraoka, N.; Miyamoto, K.; Sadahiro, T.; Isomi, M.; Haginiwa, S.; Kojima, H.; Umei, T.; Akiyama, M.; Kuishi, Y., et al. Fibroblast Growth Factors and Vascular Endothelial Growth Factor Promote Cardiac Reprogramming under Defined Conditions. Stem cell reports 2015, 5, 1128-1142, doi:10.1016/j.stemcr.2015.10.019.

21. Mohamed, T.M.A.; Stone, N.R.; Berry, E.C.; Radzinsky, E.; Huang, Y.; Pratt, K.; Ang, Y.-S.; Yu, P.; Wang, H.; Tang, S., et al. Chemical Enhancement of In Vitro and In Vivo Direct Cardiac ReprogrammingClinical Perspective. Circulation 2016, 135, 978-995, doi:10.1161/CIRCULATIONAHA.116.024692.

22. Reichardt, I.M.; Robeson, K.Z.; Regnier, M.; Davis, J. Controlling cardiac fibrosis through fibroblast state space modulation. Cell Signal 2021, 79, 109888, doi:10.1016/j.cellsig.2020.109888.

23. Fu, X.; Khalil, H.; Kanisicak, O.; Boyer, J.G.; Vagnozzi, R.J.; Maliken, B.D.; Sargent, M.A.; Prasad, V.; Valiente-Alandi, I.; Blaxall, B.C., et al. Specialized fibroblast differentiated states underlie scar formation in the infarcted mouse heart. J Clin Invest 2018, 128, 2127-2143, doi:10.1172/Jci98215. 
24. Zhou, Y.; Liu, Z.; Welch, J.D.; Gao, X.; Wang, L.; Garbutt, T.; Keepers, B.; Ma, H.; Prins, J.F.; Shen, W., et al. Single-Cell Transcriptomic Analyses of Cell Fate Transitions during Human Cardiac Reprogramming. Cell Stem Cell 2019, https://doi.org/10.1016/j.stem.2019.05.020, doi:https://doi.org/10.1016/j.stem.2019.05.020.

25. Liu, Z.; Wang, L.; Welch, J.D.; Ma, H.; Zhou, Y.; Vaseghi, H.R.; Yu, S.; Wall, J.B.; Alimohamadi, S.; Zheng, M., et al. Single-cell transcriptomics reconstructs fate conversion from fibroblast to cardiomyocyte. Nature 2017, 10.1038/nature24454, doi:10.1038/nature24454.

26. Carthy, J.M. TGFbeta signaling and the control of myofibroblast differentiation: Implications for chronic inflammatory disorders. J Cell Physiol 2018, 233, 98-106, doi:10.1002/jcp.25879.

27. Zhao, Y.; Londono, P.; Cao, Y.; Sharpe, E.J.; Proenza, C.; O'Rourke, R.; Jones, K.L.; Jeong, M.Y.; Walker, L.A.; Buttrick, P.M., et al. High-efficiency reprogramming of fibroblasts into cardiomyocytes requires suppression of pro-fibrotic signalling. Nat Commun 2015, 6, 8243, doi:10.1038/ncomms9243.

28. Riching, A.S.; Danis, E.; Zhao, Y.; Cao, Y.; Chi, C.; Bagchi, R.A.; Klein, B.J.; Xu, H.; Kutateladze, T.G.; McKinsey, T.A., et al. Suppression of canonical TGF-beta signaling enables GATA4 to interact with H3K27me3 demethylase JMJD3 to promote cardiomyogenesis. J Mol Cell Cardiol 2020, 153, 44-59, doi:10.1016/j.yjmcc.2020.12.005.

29. Bektik, E.; Fu, J.D. Production of Cardiomyocyte-Like Cells by Fibroblast Reprogramming with Defined Factors. Methods Mol Biol 2021, 2239, 33-46, doi:10.1007/978-1-0716-1084-8_3.

30. Walraven, M.; Akershoek, J.J.; Beelen, R.H.; Ulrich, M.M. In vitro cultured fetal fibroblasts have myofibroblast-associated characteristics and produce a fibrotic-like environment upon stimulation with TGF-beta1: Is there a thin line between fetal scarless healing and fibrosis? Arch Dermatol Res 2017, 309, 111-121, doi:10.1007/s00403-016-1710-3.

31. Santiago, J.J.; Dangerfield, A.L.; Rattan, S.G.; Bathe, K.L.; Cunnington, R.H.; Raizman, J.E.; Bedosky, K.M.; Freed, D.H.; Kardami, E.; Dixon, I.M. Cardiac fibroblast to myofibroblast differentiation in vivo and in vitro: expression of focal adhesion components in neonatal and adult rat ventricular myofibroblasts. Dev Dyn 2010, 239, 1573-1584, doi:10.1002/dvdy.22280.

32. Srivastava, D.; Ieda, M. Critical factors for cardiac reprogramming. Circ Res 2012, 111, 5-8, doi:10.1161/CIRCRESAHA.112.271452.

33. Takeda, J.; Adachi, K.; Halprin, K.M.; Itami, S.; Levine, V.; Woodyard, C. Forskolin activates adenylate cyclase activity and inhibits mitosis in in vitro in pig epidermis. J Invest Dermatol 1983, 81, 236-240, doi:10.1111/1523-1747.ep12518219.

34. Seamon, K.B.; Padgett, W.; Daly, J.W. Forskolin: unique diterpene activator of adenylate cyclase in membranes and in intact cells. Proc Natl Acad Sci U S A 1981, 78, 3363-3367, doi:10.1073/pnas.78.6.3363.

35. Kockskamper, J.; Erlenkamp, S.; Glitsch, H.G. Activation of the cAMP-protein kinase A pathway facilitates Na+ translocation by the Na+-K+ pump in guinea-pig ventricular myocytes. J Physiol 2000, 523 Pt 3, 561-574, doi:10.1111/j.1469-7793.2000.t01-2-00561.x.

36. Delghandi, M.P.; Johannessen, M.; Moens, U. The cAMP signalling pathway activates CREB through PKA, p38 and MSK1 in NIH 3T3 cells. Cell Signal 2005, 17, 1343-1351, doi:10.1016/j.cellsig.2005.02.003.

37. Ge, Y.C.; Li, J.N.; Ni, X.T.; Guo, C.M.; Wang, W.S.; Duan, T.; Sun, K. Cross talk between cAMP and p38 MAPK pathways in the induction of leptin by hCG in human placental syncytiotrophoblasts. Reproduction 2011, 142, 369-375, doi:10.1530/REP-11-0053.

38. Bollong, M.J.; Yang, B.; Vergani, N.; Beyer, B.A.; Chin, E.N.; Zambaldo, C.; Wang, D.; Chatterjee, A.K.; Lairson, L.L.; Schultz, P.G. Small molecule-mediated inhibition of myofibroblast transdifferentiation for the treatment of fibrosis. Proc Natl Acad Sci U S A 2017, 114, 4679-4684, doi:10.1073/pnas.1702750114.

39. Wen, J.; Lin, X.; Gao, W.; Qu, B.; Ling, Y.; Liu, R.; Yu, M. MEK inhibition prevents TGFbeta1induced myofibroblast transdifferentiation in human tenon fibroblasts. Mol Med Rep 2019, 19, 468-476, doi:10.3892/mmr.2018.9673. 
40. Swaney, J.S.; Roth, D.M.; Olson, E.R.; Naugle, J.E.; Meszaros, J.G.; Insel, P.A. Inhibition of cardiac myofibroblast formation and collagen synthesis by activation and overexpression of adenylyl cyclase. Proc Natl Acad Sci U S A 2005, 102, 437-442, doi:10.1073/pnas.0408704102.

41. Liu, X.; Sun, S.Q.; Hassid, A.; Ostrom, R.S. cAMP inhibits transforming growth factor-beta-stimulated collagen synthesis via inhibition of extracellular signal-regulated kinase 1/2 and Smad signaling in cardiac fibroblasts. Mol Pharmacol 2006, 70, 1992-2003, doi:10.1124/mol.106.028951.

42. Fu, Y.; Huang, C.; Xu, X.; Gu, H.; Ye, Y.; Jiang, C.; Qiu, Z.; Xie, X. Direct reprogramming of mouse fibroblasts into cardiomyocytes with chemical cocktails. Cell research 2015, 25, 1013-1024, doi:10.1038/cr.2015.99.

43. Kim, J.S.; Hong, Y.J.; Choi, H.W.; Choi, S.; Do, J.T. Protein Kinase A Signaling Is Inhibitory for Reprogramming into Pluripotent Stem Cells. Stem Cells Dev 2016, 25, 378-385, doi:10.1089/scd.2015.0333.

44. Li, L.; Fan, D.; Wang, C.; Wang, J.Y.; Cui, X.B.; Wu, D.; Zhou, Y.; Wu, L.L. Angiotensin II increases periostin expression via Ras/p38 MAPK/CREB and ERK1/2/TGF-beta1 pathways in cardiac fibroblasts. Cardiovasc Res 2011, 91, 80-89, doi:10.1093/cvr/cvr067.

45. Husse, B.; Isenberg, G. CREB expression in cardiac fibroblasts and CREM expression in ventricular myocytes. Biochem Biophys Res Commun 2005, 334, 1260-1265, doi:10.1016/j.bbrc.2005.06.206.

46. Husse, B.; Isenberg, G. Cyclic mechanical strain causes cAMP-response element binding protein activation by different pathways in cardiac fibroblasts. Heart Int 2010, 5, e3, doi:10.4081/hi.2010.e3.

47. Deng, X.; Deng, L.; Wang, P.; Cheng, C.; Xu, K. Posttranslational modification of CREB1 decreases collagen I expression by inhibiting the TGFbeta1 signaling pathway in rat hepatic stellate cells. Mol Med Rep 2016, 14, 5751-5759, doi:10.3892/mmr.2016.5926.

48. Liu, Y.; Xu, H.; Geng, Y.; Xu, D.; Zhang, L.; Yang, Y.; Wei, Z.; Zhang, B.; Li, S.; Gao, X., et al. Dibutyryl-cAMP attenuates pulmonary fibrosis by blocking myofibroblast differentiation via PKA/CREB/CBP signaling in rats with silicosis. Respir Res 2017, 18, 38, doi:10.1186/s12931-017-0523-z.

49. Chakraborty, D.; Zhu, H.; Jungel, A.; Summa, L.; Li, Y.N.; Matei, A.E.; Zhou, X.; Huang, J.; Trinh-Minh, T.; Chen, C.W., et al. Fibroblast growth factor receptor 3 activates a network of profibrotic signaling pathways to promote fibrosis in systemic sclerosis. Sci Transl Med 2020, 12, doi:10.1126/scitranslmed.aaz5506.

50. Ichiki, T. Role of cAMP response element binding protein in cardiovascular remodeling: good, bad, or both? Arterioscler Thromb Vasc Biol 2006, 26, 449-455, doi:10.1161/01.ATV.0000196747.79349.d1.

51. Watson, P.A.; Reusch, J.E.; McCune, S.A.; Leinwand, L.A.; Luckey, S.W.; Konhilas, J.P.; Brown, D.A.; Chicco, A.J.; Sparagna, G.C.; Long, C.S., et al. Restoration of CREB function is linked to completion and stabilization of adaptive cardiac hypertrophy in response to exercise. Am J Physiol Heart Circ Physiol 2007, 293, H246-259, doi:10.1152/ajpheart.00734.2006.

52. Watson, P.A.; Birdsey, N.; Huggins, G.S.; Svensson, E.; Heppe, D.; Knaub, L. Cardiac-specific overexpression of dominant-negative CREB leads to increased mortality and mitochondrial dysfunction in female mice. Am J Physiol Heart Circ Physiol 2010, 299, H2056-2068, doi:10.1152/ajpheart.00394.2010.

53. Molkentin, J.D.; Bugg, D.; Ghearing, N.; Dorn, L.E.; Kim, P.; Sargent, M.A.; Gunaje, J.; Otsu, K.; Davis, J. Fibroblast-Specific Genetic Manipulation of p38 Mitogen-Activated Protein Kinase In Vivo Reveals Its Central Regulatory Role in Fibrosis. Circulation 2017, 136, 549-561, doi:10.1161/CIRCULATIONAHA.116.026238.

54. Meyer-Ter-Vehn, T.; Gebhardt, S.; Sebald, W.; Buttmann, M.; Grehn, F.; Schlunck, G.; Knaus, P. p38 inhibitors prevent TGF-beta-induced myofibroblast transdifferentiation in human tenon fibroblasts. Invest Ophthalmol Vis Sci 2006, 47, 1500-1509, doi:10.1167/iovs.05-0361. 
55. Kamiya, K.; Sakakibara, K.; Ryer, E.J.; Hom, R.P.; Leof, E.B.; Kent, K.C.; Liu, B. Phosphorylation of the cyclic AMP response element binding protein mediates transforming growth factor beta-induced downregulation of cyclin $\mathrm{A}$ in vascular smooth muscle cells. Mol Cell Biol 2007, 27, 3489-3498, doi:10.1128/MCB.00665-06.

56. Lee, J.W.; Park, H.S.; Park, S.A.; Ryu, S.H.; Meng, W.; Jurgensmeier, J.M.; Kurie, J.M.; Hong, W.K.; Boyer, J.L.; Herbst, R.S., et al. A Novel Small-Molecule Inhibitor Targeting CREB-CBP Complex Possesses Anti-Cancer Effects along with Cell Cycle Regulation, Autophagy Suppression and Endoplasmic Reticulum Stress. PLoS One 2015, 10, e0122628, doi:10.1371/journal.pone.0122628.

57. Chen, P.; Li, M.; Hao, Q.; Zhao, X.; Hu, T. Targeting the overexpressed CREB inhibits esophageal squamous cell carcinoma cell growth. Oncol Rep 2018, 39, 1369-1377, doi:10.3892/or.2017.6167. 